\title{
The pathogenesis of pneumatic tourniquet paralysis in man
}

\author{
STEPHEN K YATES, LAWRENCE N HURST, WILLIAM F BROWN \\ From the Department of Clinical Neurological Sciences, University Hospital, London, Ontario, Canada
}

SUMMARY The relative importance of ischaemic and direct mechanical injury to nerves compressed by a tourniquet, in the pathogenesis of tourniquet paralysis in man has not been established. To investigate this question, conduction in ulnar or median nerve fibres has been measured in healthy subjects both at the level of the pneumatic tourniquet and distal to the tourniquet. Measurement was prior to, for the period of tourniquet inflation, and following release of the tourniquet. The earliest conduction delays and block were observed at the level of the tourniquet, particularly across the proximal tourniquet border zone. However, a proximal to distal progression in conduction abnormalities distal to the tourniquet suggested that the earlier conduction abnormalities at the level of the tourniquet were primarily ischaemic in origin. Mechanical compression, however, probably contributed to disproportionate conduction delays and blocks across the border zones of the tourniquet.

The pneumatic tourniquet is commonly employed by surgeons to provide a bloodless surgical field in the limbs. The complication rate at the University Hospital in London, Ontario has been estimated to be 1 in 5,000 or less. Rarely, paralysis, loss of sensation, or autonomic abnormalities may persist for periods of several months, even when the tourniquet is employed at recommended pressure levels and inflation times. ${ }^{1-3}$

Local conduction delays have been observed in peripheral nerves at the level of the tourniquet border zone in patients with post-tourniquet paralysis. ${ }^{3}$ These local conduction abnormalities may be the functional equivalent of the structural abnormalities observed at the proximal-distal border zones in experimental tourniquet lesions. ${ }^{4}$ These occurred at inflation times and pressures clearly beyond those employed in man. It is not known, however, what the relative importance of ischaemia and compression are to the abnormalities in nerve function at the level of the tourniquet, particularly at pressure levels and inflation times normally employed by surgeons. Experiments were therefore devised in an attempt to quantitate the relative importance of ischaemia and mechanical compression to the

Address for reprint requests: Dr WF Brown, University Hospital, 339 Windermere Rd, London, Ontario, Canada, N6A 5A5.

Accepted 8 June 1981 abnormalities in nerve function during and in the early period following tourniquet inflation in man.

\section{Methods}

These investigations were carried out during mammoplastic reductions. The protocol was reviewed and approved by the human experimentation committee at the University of Western Ontario. Informed consent was obtained from the patients. The operations were carried out under general anaesthesia but without the use of neuromuscular blocking agents. The Kidde Model 400 pneumatic tourniquet was employed ( $80 \mathrm{~mm}$ wide). This tourniquet employs a plastic cuff insert external to the inflatable cuff. The maximum inflation pressure employed was $300 \mathrm{~mm} \mathrm{Hg}$. Tourniquet inflation times varied between $\frac{1}{2}-1$ hour. Special care was taken to avoid wrinkling the skin beneath the tourniquet. For most of the experiments only one tourniquet was employed, centred at the level of the mid-upper arm, but in later experiments two tourniquets were employed, one at the level of the mid-forearm and the other about the upper arm.

\section{Electrophysiological methods}

The majority of the experiments were carried out on the ulnar nerve-hypothenar muscle complex (fig 1). The ulnar nerve was stimulated at the level of the wrist and proximal and distal to the upper arm tourniquet in the earliest experiments. Later, extra stimulating electrodes under the tourniquet made it possible to measure the conduction times across the sub-tourniquet and proximal and distal tourniquet border zones (fig 3). For these later investigations, a special multi-electrode was constructed 


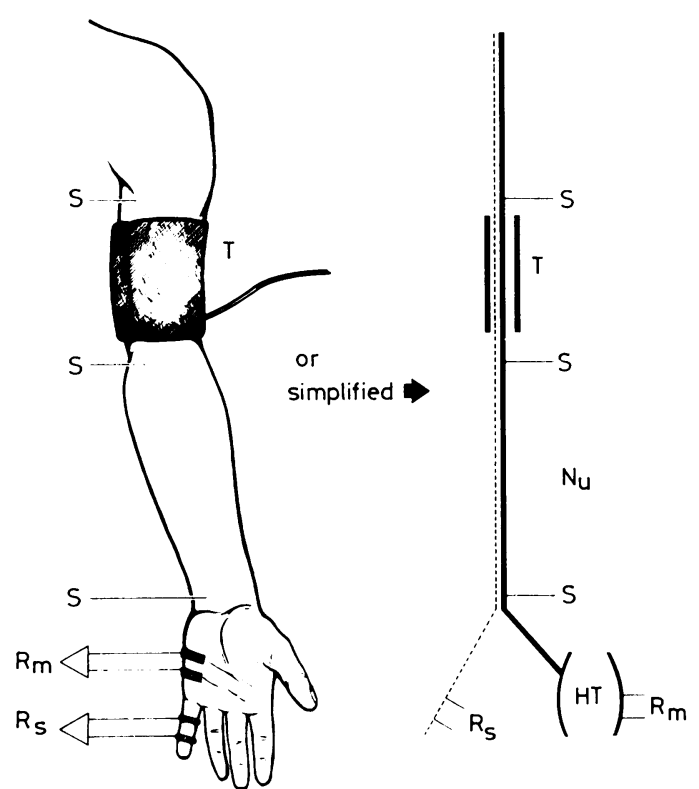

Fig 1 Left: The general arrangement of the electrodes and the position of the pneumatic tourniquet about the upper arm. Right: Corresponding schematic outline of the relative electrode positions and the tourniquet.

$S=$ Stimulating electrode (cathode) positions, $R_{m}=$ Recording electrodes (surface) over hypothenar muscle group, $R_{s}=$ Recording electrodes (DISA I3L69) positions about the Vth digit $\left(G_{1}\right.$ proximal $), T=$ Tourniquet, $N_{u}=$ Nerve-ulnar, $H T=$ Hypothenar.

which incorporated $4 \times 10 \mathrm{~mm}$ silver strip electrodes on the inner surface. The interelectrode distance across the tourniquet border zones was $50 \mathrm{~mm}$ and between the sub-tourniquet electrodes, $30 \mathrm{~mm}$. A large silver plate over the upper back was employed as the anode. In the early experiments needle electrodes were used to stimulate the ulnar or median nerves proximal and distal to the tourniquet but these were replaced by surface electrodes in the majority of the subsequent investigations. In all these experiments special care was taken to ensure that the brief $(0.1 \mathrm{~ms}$ or less) isolated stimulus pulses were just supramaximal and that the stimulating electrodes were placed directly over the respective test nerve. The latter was achieved by placing the electrodes where the maximum response was obtained at the least stimulus intensity. These criteria were rechecked after the tourniquet was placed about the forearm and in the early period following tourniquet inflation to minimise shifts in the location of the stimulating electrodes in relation to the nerve.

The temperature of the limb distal to the tourniquet was monitored by an intra-muscular thermistor electrode. Once all electrodes were in place, the limb was insulated by cotton wool, etc, to reduce the heat loss and thereby minimise the conduction changes which could result from cooling of the nerve and muscles. Testing was carried out prior to tourniquet inflation, at five minute intervals or less following tourniquet inflation and continued for up to one hour after tourniquet release. The least conduction times (or conversely the maximum conduction velocities) and the degree of conduction block were measured across the level of the tourniquet and distal to the tourniquet. the latter in the pure ischaemic zone. The " $M$ " response was recorded by surface electrodes. The position of the stigmatic electrode was adjusted to obtain the maximum peak-to-peak voltage $(p-p V)$, least rise time and initial negative deflection for the thenar (T) or hypothenar (HT) muscle groups innervated by the respective ulnar or median nerves. The degree of conduction block was estimated by changes in the maximum " $M$ " response p-pV or area. Area was measured only in the earliest experiments because it was learned that there was little change in $\mathrm{p}-\mathrm{pV}$ duration in the " $M$ " response, that is, little evidence of temporal dispersion in the " $M$ " response (figs 2,3 ).

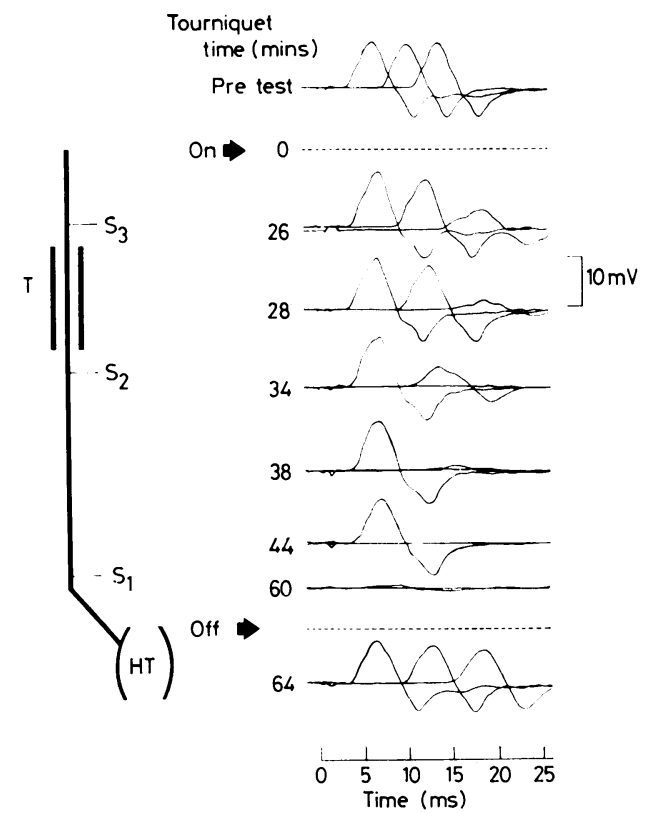

Fig 2 Left: Schematic of the relative positions of the stimulating electrodes at the levels of the wrist $\left(S_{1}\right)$ just distal to the tourniquet $\left(S_{2}\right)$ and proximal to the tourniquet $\left(S_{3}\right)$ about the mid-upper arm. Right: Selected records to illustrate the changes in the hypothenar $(H T)$ " $M$ " response to stimulation at the $S_{1}, S_{2}$ and $S_{3}$ levels at various times prior to and after tourniquet inflation and following release of the tourniquet. Note (1) the earliest conduction block across the tourniquet segment (between $S_{2}$ and $S_{3}$ ), (2) persistence of the " $M$ " response evoked by stimulation at $S_{1}$, (3) the overall cascade beginning at the most proximal level of stimulation and moving toward the most distal stimulation level and (4) the relative degree of conduction block and delay. 


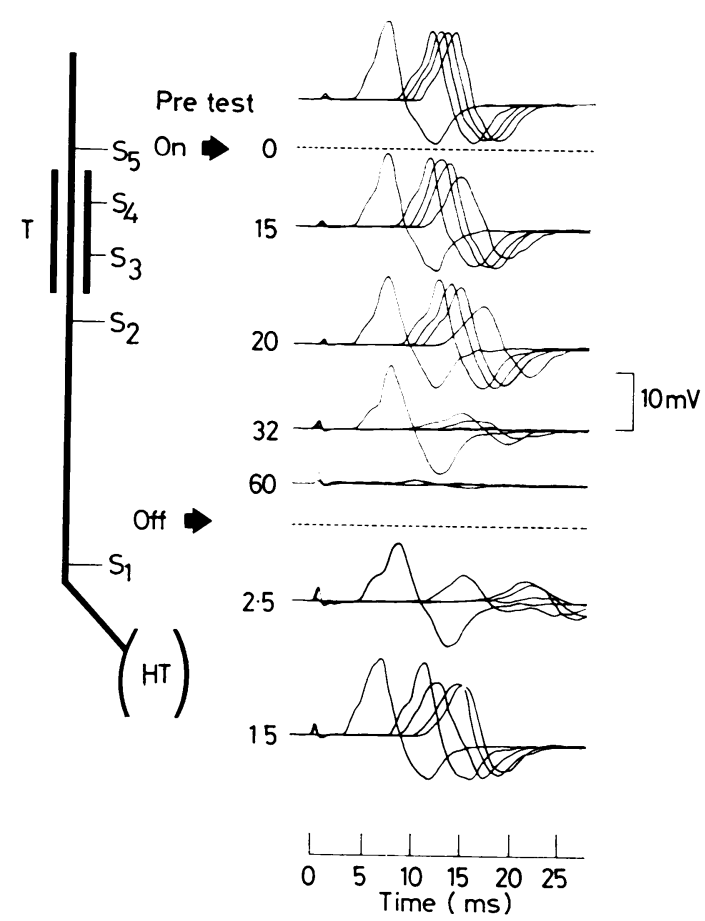

Fig 3 Left: Schematic of the relative positions of the stimulating electrodes including $S_{1}$ at the level of the wrist, $S_{2}$ just distal to the tourniquet, $S_{3}$ and $S_{4}$ beneath the tourniquet and $S_{5}$ iust proximal to the tourniquet. Tourniquet about the mid-upper arm. Right: The changes in the hypothenar $(H T)$ " $M$ " response at various times after tourniquet inflation and release in relation to the pre-test observations. Note (1) the earliest conduction block across the proximal tourniquet border zone, (2) the early disproportionate conduction delay across the distal tourniquet border zone in relation to the subtourniquet segment, (3) the overall proximal to distal cascade in the degree of conduction block and (4) following tourniquet release, the disproportionate conduction delay across the distal tourniquet border zone at 2.5 minutes and persistent conduction block across the segment even at 15 minutes.

\section{Results}

(1) THE RELATIVE CHANGES IN CONDUCTION ACROSS THE LEVEL OF THE TOURNIQUET AND DISTAL TO THE TOURNIQUET

The earliest conduction block was observed across the level of the tourniquet at 10-20 minutes in HT motor fibres (fig 2). Only later, between 20-30 minutes, was conduction block evident distal to the tourniquet and only by 40 minutes over the most distal segment, beyond the level of the wrist. The largest relative increase in conduction time was observed across the tourniquet zone. Equivalent reductions in the maximum conduction velocity were observed only later, distal to the tourniquet; corresponding increases in the motor terminal latency being delayed the longest (figs 2,3 ). Just prior to complete impulse block, the maximum conduction velocity (MCV) was less than one half the pre-tourniquet values. These reductions were not accounted for by lower temperatures in the arm because only a 1-3 degree centigrade loss was observed provided the limb was properly insulated.

(2) CHANGES IN CONDUCTION ACROSS THE SUB-TOURNIQUET SEGMENT RELATIVE TO THE PROXIMAL AND DISTAL TOURNIQUET BORDER ZONES

\section{(i) Motor fibres}

In HT motor fibres the earliest (less than 15 minutes) and largest increases in conduction time were observed across the proximal tourniquet border zone (figs 3 and 4A-B). In some subjects (fig 3) relatively larger increases in the conduction time across the distal tourniquet border zone in relation to the sub-tourniquet segment were observed. This was not observed in all subjects (fig 4). Conduction block was evident earlier (15-20 minutes) and was first complete across the proximal tourniquet border zone. The observation that little or no change in $p-p$ duration accompanied the reduction in the $p-p V$ meant that it was unlikely the latter was a product, in part, of temporal dispersion of the " $M$ " response or preferential block of the fastest or slowest conduction velocity fibres.

\section{(ii) Sensory fibres}

The earliest and maximum conduction blocks were observed across the tourniquet border zones, particularly the proximal border zone. This was evident within less than 10 minutes of tourniquet inflation. Because of the shorter duration of single nerve fibre action potentials (SNAP) $(<5 \mathrm{~ms})$ compared to motor unit potentials $(10-20 \mathrm{~ms})$ and the lower relative voltage $(<0.1 \mathrm{mV})$ of the maximum SNAP relative to even a single motor unit potential $(<0.5 \mathrm{mV})$ it was much harder to quantitate the contribution of temporal dispersion to reductions in the SNAP p-pV. However, whatever the relative contribution of conduction block and temporal dispersion to the observed changes, a disproportionate reduction in the SNAP $\mathrm{p}-\mathrm{pV}$ across the tourniquet border zones in relation to the sub-tourniquet segment was observed in the first 20 minutes. 


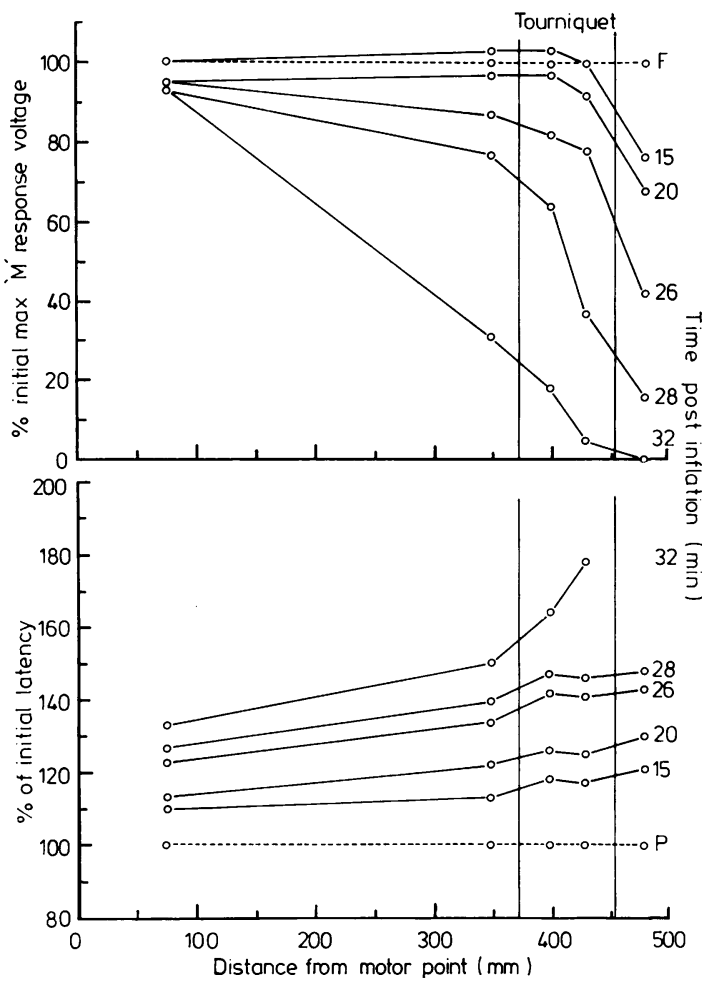

Fig 4 Electrode locations relative to an upper arm tourniquet idential to fig 3. Upper: Plot of the percentage changes at various times (in minutes) in the maximum hypothenar " $M$ " response (compared to the initial values) evoked by stimulation of the ulnar nerve proximal, beneath and distal to the tourniquet. Lower: Corresponding percentage changes in least latencies of the hypothenar " $M$ " response evoked by stimulation at the various levels compared to their respective initial values. The parallel vertical lines illustrate the positions of the proximal and distal tourniquet border zones in relation to the electrode positions. The distance from the hypothenar motor point to the stimulating electrode (in $\mathrm{mm}$ ) is plotted on the $X$ axis. Note (1) the early disproportionate conduction delays across the tourniquet border zones and (2) the earliest conduction block across the proximal, and in this patient, sub-tourniquet zones.

\section{(3) PROXIMAL TO DISTAL PROGRESSION} IN THE CONDUCTION ABNORMALITIES

The above observations established that the earliest and maximum functional abnormalities were observed at the level of the tourniquet; specifically across the proximal tourniquet border zone. These observations were initially interpreted to indicate a primary mechanical rather than ischaemic factor in the pathogenesis of the functional abnormalities at the level of the tourniquet. It was obvious, however,

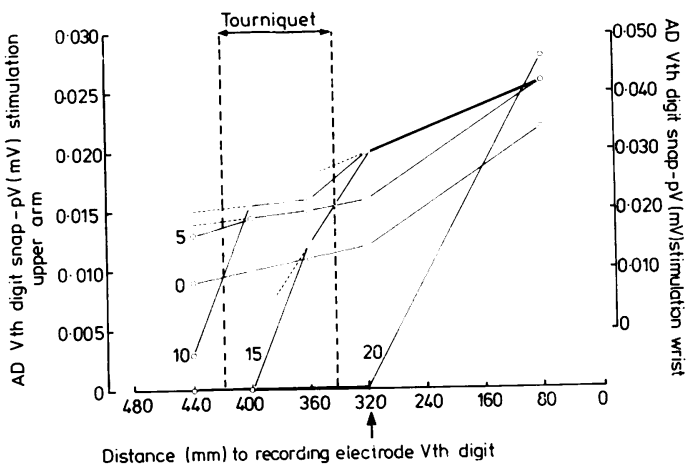

Fig 5 Schematic as per fig 1. Plot of the negative peak voltage $(-p V)$ in $m V$ of the antidromic fifth digit sensory nerve action potential ( $A D$ Vth Digit $S N A P)$ at various times expressed in minutes following inflation of the pneumatic tourniquet about the upper arm. Ulnar nerve stimulation was carried out at four levels in the upper arm to measure the changes in the SNAP $-p V$ across the proximal and distal tourniquet border zones and across the sub-tourniquet segment. The ulnar nerve was also stimulated at the level of the wrist. The position of the tourniquet border zone relative to the stimulating electrodes is shown by the interrupted vertical lines. Note (1) the different voltage scales for the SNAP values in response to stimulation at the wrist and the other stimulation points at the upper arm, (2) the distance between the recording electrode $\left(G_{1}\right)$ and the stimulating cathode is shown on the $X$ axis in $\mathrm{mm}$. The scale factor is changed at $320 \mathrm{~mm}$. The earliest reduction in SNAP voltage was observed at 5 min across the proximal tourniquet border zone and disproportionate reductions in SNAP voltage were obvious across both tourniquet zones by $10 \mathrm{~min}$. The interrupted extensions represent what the expected changes with distance might have been without conduction block or temporal dispersion. By 15 minutes there was complete block across the sub-tourniquet and proximal border zone segments.

that there was a proximal to distal progression in the increases in conduction time and conduction block in the ischaemic zone distal to the tourniquet in both motor and sensory fibres. For example, the conduction time increases and magnitude of the conduction block at equivalent ischaemic times were more abnormal across the forearm than in the terminal wrist to muscle segment (figs $1,2,3,4)$. To illustrate: in fig 2, at 28 minutes the increase in conduction time across the forearm segment was $22 \%$ but there was only a $13 \%$ increase in the motor terminal latency, the corresponding increase in conduction time across the tourniquet segment being $53 \%$. There was, therefore, evidence for a proximal to distal progression in the degree of conduction block and conduction delay both in motor and sensory fibres. This in turn implied that the degree of con- 

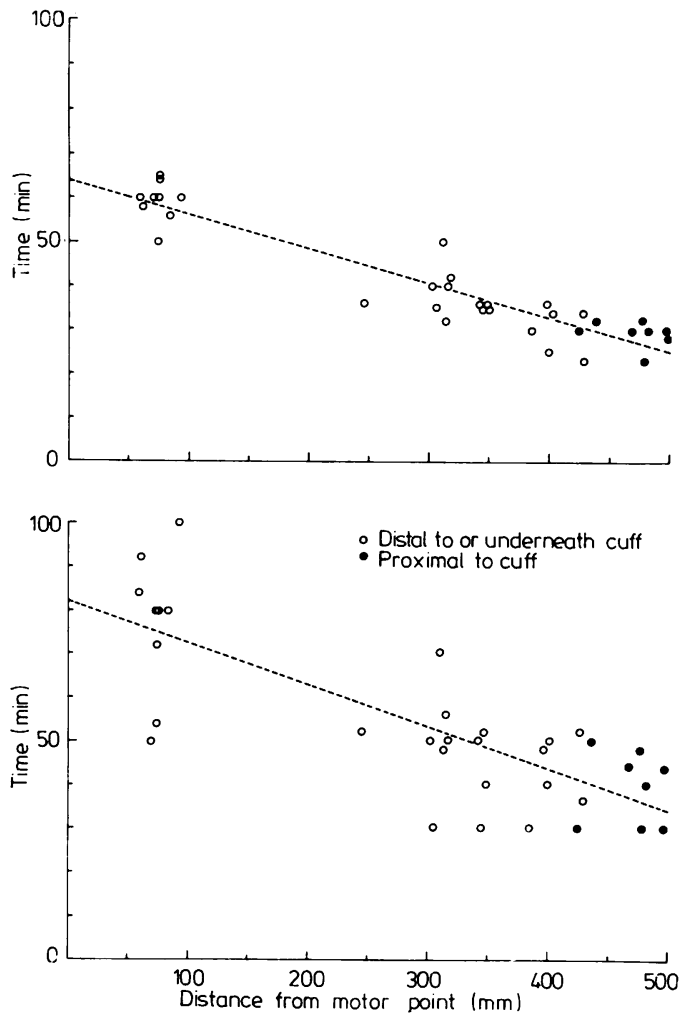

Fig 6 Plots of the relative times to initial conduction block (upper) and complete conduction block (lower) in the hypothenar " $M$ " response at various distances proximal to the hypothenar motor point. Stimulation points proximal to the pneumatic tourniquet are distinguished by the solid circles. The plots represent a combination of observations from eight patients.

The time to the earliest conduction block and complete conduction block was a function of the distance between the point of stimulation and the motor point.

duction block and conduction delay was related to the ischaemic length of the nerve between the point of stimulation and the stigmatic electrode (fig 6). This was substantiated by the observation that the time to $50 \%$ conduction block in HT motor fibres when the ulnar nerve was stimulated just proximal to an upper limb tourniquet was 20-25 minutes but when the ulnar nerve was stimulated just proximal to a forearm tourniquet, the time to corresponding block was longer, namely 30-40 minutes.

Further evidence to support the importance of nerve length as a determinant of the time to conduction block was provided by tests on which the times to equivalent block were compared in HT and flexor carpi ulnaris (FCU) motor fibres in response to stimulation proximal to an upper arm tourniquet.

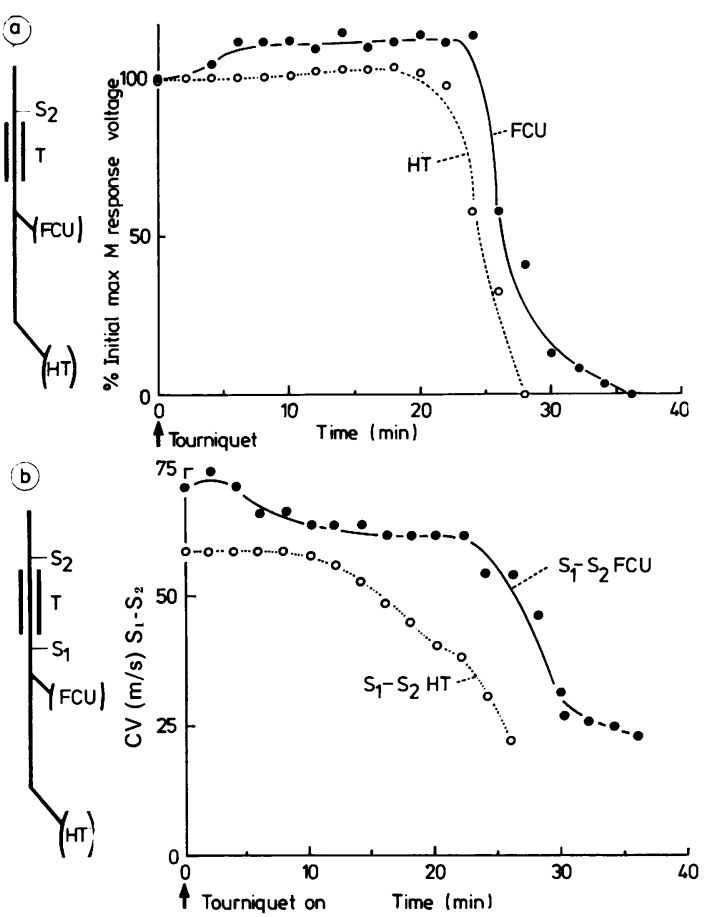

Fig 7 Upper: Left-schematic illustration of the relative positions of the recording electrodes (surface) over the flexor carpi ulnaris (FCU) and hypothenar (HT) muscles in relation to the pneumatic tourniquet about the mid-upper arm and the stimulating electrode; located just proximal to the tourniquet. Right-plot of the changes in the respective $F C U$ and $H T$ " $M$ " response maximum $p-p V$ compared to their initial values at the various times after tourniquet inflation. Lower: Leftrelative positions of the stimulating and recording electrodes in relation to the tourniquet are the same as above except for the inclusion of an extra stimulating electrode $\left(S_{1}\right)$ just distal to the tourniquet border zone. Right-changes in the maximum conduction velocity $\mathrm{m} / \mathrm{s}$ in flexor carpi ulnaris (FCU) and hypothenar (HT) motor fibres across the segment $S_{1}-S_{2}$. Note the larger degree of conduction block and greater reduction in the maximum conduction velocity in hypothenar motor fibres at corresponding times following tourniquet inflation.

Not only was the time to block longer for the shorter FCU fibres but the reduction in conduction velocity was much larger for the HT compared to the FCU fibres across the tourniquet segment at equivalent times after tourniquet inflation. The above evidence therefore established that the time to the earliest detection of abnormalities in conduction and the degree of those functional abnormalities were a function of the length of the nerve in the ischaemic 
zone. The shorter the length of the nerve tested in the ischaemic territory, the longer was the time to conduction block and the less the degree of conduction delay.

The ischaemic zone included both the segment beneath the tourniquet and the zone distal to the tourniquet. Therefore, it was possible that the same factor which accounted for the proximal to distal progression in the degree of abnormality in nerve function which was observed distal to the tourniquet accounted also for the even earlier conduction abnormalities at the level of the tourniquet border zone. With the methods employed, the last represented the longest accumulative length of nerve tested. This led to a fundamental question. If the proximal to distal progression in functional abnormalities observed was a function of ischaemia alone, were there any abnormalities which could be directly related to mechanical compression?

(4) EVIDENCE FOR MECHANICAL COMPRESSION

To establish mechanical compression as a factor required that abnormalities in nerve function be observed beneath or across the tourniquet border zones which could not be explained by the orderly proximal-to-distal progression in abnormal nerve function observed in the pure ischaemic zone distal to the tourniquet. Disproportionately larger conduction delays and block would have to be demonstrated across the distal tourniquet border zone compared to the more proximal sub-tourniquet segment. This was indeed evident in some subjects in both motor and sensory fibres within the first 30 minutes (figs 3,5 ). It was not, however, possible to demonstrate conduction abnormalities across the tourniquet border zones which were not accompanied by other parallel, though less severe, conduction changes distal to the tourniquet. This meant that whatever mechanical pressure factors were operative at the level of the tourniquet, these factors were likely to be overtaken by the general ischaemic changes. The mechanical pressure factor was perhaps more obvious in the post-tourniquet release period when, at an early stage, a disproportionate increase in conduction time and block was very obvious across the distal tourniquet border zones compared to the more proximal sub-tourniquet and proximal tourniquet segments (fig 3). Even at 1 hour, conduction delays were commonly present across the tourniquet segment when there had been $100 \%$ recovery distal to the tourniquet. In order to look for a distinct pressure factor an attempt was made to separate the possible ischaemic and pressure factors (fig 8). In this test two tourniquets were employed, one about the mid-forearm and the other about the

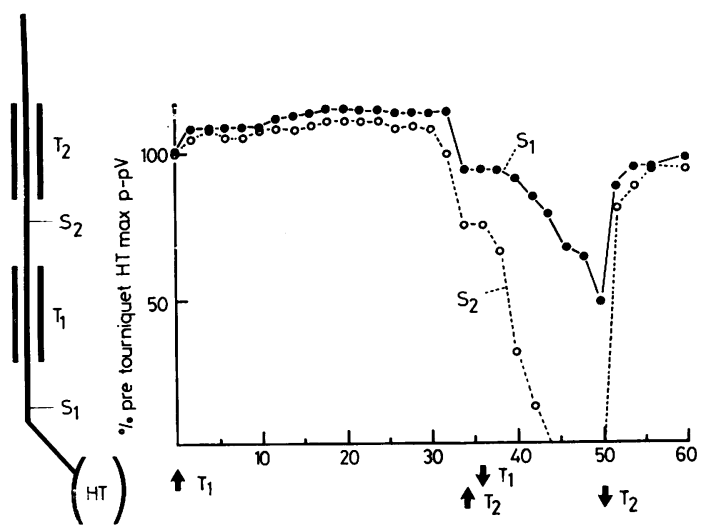

Fig 8 Left: The relative positions of the stimulating electrodes, $S_{1}$ at the level of the wrist and $S_{2}$ at the level of the elbow compared to the two tourniquets, $T_{1}$ about the mid-forearm and $T_{2}$ about the mid-upper arm. Right: Changes in the maximum hypothenar " $M$ " response $p-p V$ compared to the initial pre-tourniquet values at various times following tourniquet inflation in response to stimulation at the $S_{1}$ and $S_{2}$ levels. At time 0 $\mathrm{T}_{1}$ was inflated to $300 \mathrm{~mm} \mathrm{Hg}$. When conduction block was clearly established in response to stimulation at $S_{2}$ the upper arm tourniquet was inflated $\left(T_{2}\right)$ and shortly thereafter, the forearm tourniquet $\left(T_{1}\right)$ was released. The upper arm tourniquet $\left(T_{2}\right)$ was finally released at 50 min. Note, no reversal in the conduction block across the forearm was observed even in the initial period following release of the forearm tourniquet.

mid-upper arm. $\mathrm{T}_{1}$ was inflated first. When a $50 \%$ block was evident in ulnar-HT motor fibres across the forearm, $T_{2}$ was inflated and $T_{1}$ released. The primary question was whether there was any reversal of the functional abnormalities across the $\mathrm{T}_{1}$ when the mechanical compression was released though the nerve remained in the ischaemic zone. If the block in conduction had been primarily the result of mechanical compression, reversal in the block would be expected. If, however, the primary factor was ischaemia no change in the rate of progression in the block would be expected. There were mixed results. In some subjects no significant change in the rate of progression of the conduction abnormalities was observed or if present (fig 8) the temporary reduction in the rate of progression was observed both across the forearm tourniquet segment and distal to the tourniquet. This suggested that this partial recovery may have been the result of release into the forearm and hand regions of oxygenated blood trapped distal to the upper arm tourniquet. In other subjects there was a partial reversal of the conduction block observed when $T_{1}$ was released. This reversal was never complete. 
There may, therefore, be a mechanical factor but this is rapidly overtaken by the ischaemic changes.

\section{Discussion}

The primary question in this investigation was whether functional abnormalities in human nerves compressed by a tourniquet for conventional times and pressures were the result of ischaemia or mechanical compression or a combination of both factors. Ischaemia is the only factor distal to the tourniquet. The issue was, however, complicated at the level of the tourniquet where nerves were exposed to both ischaemia and mechanical compression. The distal tourniquet border zone was both compressed and ischaemic while the proximal tourniquet border zone included a proximal segment which was not ischaemic together with a distal segment which was both compressed and ischaemic beneath the tourniquet. Both border zones were subject to non-uniform pressures and stress patterns beneath the edges of the tourniquet. ${ }^{5}$ The worst combination of ischaemia, mechanical compression and non-uniform pressure distributions would therefore probably be present across the distal tourniquet border zones.

In the pure ischaemic zone distal to the tourniquet, there was evidence for a proximal to distal progression in the order of onset and severity of conduction abnormalities in the nerves. This pattern has been reported earlier. ${ }^{6}$ The proximal to distal progression may even explain the earliest conduction block and disproportionate conduction delays observed across the proximal tourniquet border zone and across the tourniquet segment as a whole, compared to the zone distal to the tourniquet. The order of progression in conduction block distal to the tourniquet was to be expected on the basis of other evidence too, on the effect of ischaemia on nerve conduction. ${ }^{78}$ The reverse of the above pattern however, would be expected if the probability of conduction block per unit length of nerve was uniform along the length of the nerve. ${ }^{9}$ The effect of this would be for the maximum reduction in the " $M$ " response per unit length of the nerve to diminish from distal to proximal; the reverse of the pattern observed here (Appendix A, fig 9). The evidence therefore suggested that the probability of conduction per unit length of nerve was not uniform but higher over the more proximal segments of the nerve. This was substantiated by the disproportionately larger increases in conduction times over the more proximal segments of the nerve. This partially explained why at equivalent times after tourniquet inflation, the degree of conduction block was higher in the longer HT fibres than in the shorter
FCU fibres.

The actual basis for the observed proximal to distal progression in the severity of the conduction abnormalities in the ischaemic nerve has not been explained. There was no obvious reason to expect this progression if all segments of the nerve proximal or distal were exposed to the equivalent degree of ischaemia. Moreover, the opposite would be expected if there were a proximal to distal fall-off in temperature or if there was any leakage of blood past the tourniquet to tissues just beyond the cuff. The latter was unlikely because at the tourniquet pressures employed it was rare to observe any blood leakage in surgical fields beyond the tourniquet. There are no anatomical or functional characteristics of nerve known to the authors which properly explain this observed proximal to distal conduction time gradient. Hence, the primary factor to explain the abnormalities in conduction at and distal to the tourniquet was probably ischaemia. This was suggested earlier by Landau. ${ }^{10}$ It was, however, possible that mechanical pressure effects if they required a sufficiently long enough time to develop, may have been masked by the effects of ischaemia. There were indeed, certain conduction abnormalities in the early post-inflation and later release periods which pointed to probable local mechanical contributions to the observed abnormalities in nerve function particularly across the border zones of the tourniquet.

Experimental evidence has emphasised the critical importance of non-uniform pressure distributions at the border zone of the tourniquet in the pathogenesis of characteristic structural abnormalities in nerve compressed by the tourniquet. ${ }^{411} 12$ However, in these experimental preparations, the compression times and pressure levels were substantially above those employed in our human investigations. Of course it is hard to compare directly the human and animal experiments. In view, however, of the complete recovery in nerve function in our experiments, in most by 1 hour and in all by 24 hours, whatever temporary structural abnormalities may have accompanied the compression, these were reversible.

Pneumatic tourniquet injury to peripheral nerve may be more common than is appreciated even at conventionally accepted compression times and pressures. For example, denervation has been detected in lower limb muscles distal to the tourniquet in knee operations. ${ }^{13}$ Therefore, the tourniquet, even as generally employed has the potential for injury to peripheral nerves. This risk is enhanced when longer compression times or higher pressures are employed. Higher pressure is a real danger; at our own hospital, calibration of tourniquets in 
common use revealed at least three which registered pressures $50-100 \mathrm{~mm} \mathrm{Hg}$ above that which the instrument itself registered. There is a need for frequent recalibration of tourniquets and proper instructions of personnel directly responsible for their use.

The present investigations have revealed that the primary factor accounting for observed functional abnormalities in human nerves compressed by the pneumatic tourniquet is ischaemia. From this it must not be concluded that ischaemia is the primary cause of tourniquet paralysis. Indeed quite the opposite is probably true in so far as ischaemia by itself, even when it lasts much longer than compression, does not cause persistent conduction block and especially the localised conduction delays and conduction block observed across the tourniquet border zones in tourniquet paralysis. ${ }^{14}$ What is meant rather is that when compression and ischaemia are combined, as they are in the tourniquet lesion, the functional abnormalities resulting from ischaemia develop sufficiently early at the level of the tourniquet as to largely mask the direct mechanical effects. Even so, functional abnormalities attributable to pressure alone are detectable. For example, disproportionate abnormalities in nerve function at the level of the proximal and distal border zones of the tourniquet point to the probable contribution of mechanical compression as the primary factor. Whatever other compressive effects may have been present were lost in the progressive ischaemic conduction block. Therefore at the time of tourniquet compression it is hard to weigh the relative contribution of ischaemia and mechanical compression to the observed functional abnormalities in the nerve. It is clear that whatever the mechanisms responsible, the functional abnormalities quickly reverse following tourniquet release when the compression times are relatively short and the pressures not excessive. Excessive pressure or compression time may however produce conduction abnormalities localised to the border zones of the cuff which may not promptly reverse and are most likely due to mechanical compression. ${ }^{3}$

\section{APPENDIX A}

To predict the degree of conduction block evident in changes in the " $M$ " response $p-p V$ at increasing distances from the motor point a theoretical model has been constructed. The critical assumptions in the simple model include: (1) The probability of conduction block per unit length (internode length) of nerve is constant along the length of the nerve. Let this probability $=$ p. (2) Each motor unit makes the same surface voltage contributions to the " $M$ " response voltage. Let that voltage be V. (3) All motor fibre conduction velocities are the same. Also let $y$ be the number of nerve fibres in this model and let there be $n$ number of internode lengths. The model made no adjustment for an increase in temporal dispersion with an increase in distance from the motor point. Experimentally, however, there was no substantial increase in $p$-p duration of the " $M$ " response with distance from the motor point before or following tourniquet inflation.

\section{Question}

What is the degree of conduction block expressed as a percentage reduction in the " $\mathrm{M}$ " $\mathrm{p}-\mathrm{pV}$ at increasing distances between the point of stimulation and the motor point?

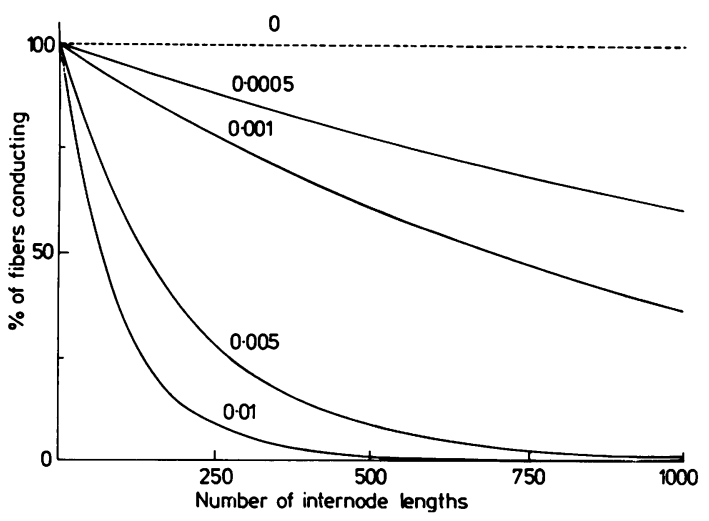

Fig 9 Plot of the percentage of nerve fibres conducting at various numbers of internode lengths away from the recording electrode at " 0 ". For the various plots the probabilities of conduction block across any one internode length are shown.

The number of fibres contributing to the "M" response at various distances from the motor point may be predicted by:

If the number of nerve fibres contributing to the " $M$ " response at distance " $O$ " (here the motor point) $=y$ and the voltage generated per nerve fibre equals $V$, then the " $M$ " response voltage at " 0 " $=y \times v$. Now let $p$ be the probability of conduction block in each fibre per unit length (here considered to be one internode length) then at the 1st internode the number of fibres conducting $=y-(y \times p)$ or $y(1-p)$.

At the 2nd internode-number of fibres conducting $=y(1-p)-y(1-p) \times p$ or $y(1-p)^{2}$

At the 3rd internode-number of fibres conducting $=y(1-p)^{2}-y(1-p)^{2} \times p$ or $\mathrm{y}(1-\mathrm{p})^{3}$ 
Finally, at the nth internode-number of nerve fibres conducting $=y(1-p)^{n}$; therefore, the voltage at distance $\mathrm{n}$ number of internodes $=$ $\mathrm{y}(1-\mathrm{p})^{\mathrm{n}} \times \mathrm{V}$.

This model predicts that the maximum reduction in p-pV per unit length of nerve will be evident over the distal segments-there being a reduction in the "apparent" degree of block with increasing distance from the motor point. This progression stood in direct contrast to the proximal to distal progression observed. Mechanical compression could explain the earliest conduction block at the level of the tourniquet. However, this seemed unlikely in view of the progression distal to the tourniquet which was observed to follow soon after the earlier changes at the level of the tourniquet. It is possible that the probability of conduction block per unit length of nerve was not uniform but increased from distal to proximal. This fitted best with the evidence.

\section{References}

${ }^{1}$ Rudge P. Tourniquet paralysis with prolonged conduction block. J Bone Joint Surg (Br) 1974;56:715-20.

${ }^{2}$ Sunderland S. Nerves and Nerve Injuries. 2nd ed. Edinburgh: Churchill Livingstone, 1978.

${ }^{3}$ Bolton CF, McFarlane RM. Human pneumatic tourniquet paralysis. Neurology (Minneap.) 1978; 28:787-93.

4 Ochoa J, Fowler TJ, Gilliatt RW. Anatomical changes in peripheral nerves compressed by a pneumatic tourniquet. J Anatomy 1972;113:433-55.

${ }^{5}$ Griffiths JC, Heywood OB. Biomechanical aspects of the tourniquet. Hand 1973;5:113-8.

${ }^{6}$ Lewis T, Pickering GW, Rothschild P. Centripetal paralysis arising out of arrested blood flow to the limb, including notes on a form of tingling. Heart $1931 ; 16: 1-32$.

7 Nielsen VK, Kardel T. Decremental conduction in normal human nerves subjected to ischemia. Acta Physiol Scand 1974;92:249-62.

${ }^{8}$ Nielsen VK. Pathophysiological aspects of uraemic neuropathy. In: Peripheral Neuropathies. North Holland Biomedical Press, Elsevier 1978.

9 Waxman SG, Brill MH, Geschwind N, Sabin TD, Lettvin JY. Probability of conduction deficit as related to fiber length in random distribution models of peripheral neuropathies. J Neurol Sci 1976;29: 39-53.

${ }^{10}$ Landau WM. Mechanism of nerve block. Nature 1972;237:224.

11 Grundfest $\mathbf{H}$. Effects of hydrostatic pressures upon the excitability, the recovery and the potential sequence of frog nerves. Cold Spring Harbour Symp Biol 1936; 4:179-87.

${ }^{12}$ Bentley FH, Schlapp W. The effect of pressure on conduction in peripheral nerve. J Physiol (Lond) 1943;102:72-82.

${ }^{13}$ Weingarden SI, Louis DL, Waylonis GW. Electromyographic changes in post meniscectomy patients: role of the pneumatic tourniquet. $J A M A 1979 ; 241$ : 1248-50.

${ }^{14}$ Hess K, Eames RA, Darveniza P, Gilliatt RW. Acute ischaemic neuropathy in the rabbit. $J$ Neurol Sci $1979 ; 44: 19-43$. 